\title{
Interoperability in eHealth Systems
}

\author{
Asuman Dogac \\ SRDC Ltd., METU Technopolis, Ankara, Turkey
}

\begin{abstract}
Interoperability in eHealth systems is important for delivering quality healthcare and reducing healthcare costs. Some of the important use cases include coordinating the care of chronic patients by enabling the co-operation of many different eHealth systems such as Electronic Health Record Systems (EHRs), Personal Health Record Systems (PHRs) and wireless medical sensor devices; enabling secondary use of EHRs for clinical research; being able to share life long EHRs among different healthcare providers. Although achieving eHealth interoperability is quite a challenge both because there are competing standards and clinical information itself is very complex, there have been a number of successful industry initiatives such as Integrating the Healthcare Enterprise (IHE) Profiles, as well as large scale deployments such as the National Health Information System of Turkey and the epSOS initiative for sharing Electronic Health Records and ePrescriptions in Europe.

This article briefly describes the subjects discussed in the VLDB 2012 tutorial to provide an overview of the issues in eHealth interoperability describing the key technologies and standards, identifying important use cases and the associated research challenges and also describing some of the large scale deployments. The aim is to foster further interest in this area.
\end{abstract}

\section{INTRODUCTION}

Interoperability is important in the eHealth domain to increase the quality of healthcare and to decrease costs. There are several real life cases that will benefit from interoperability, such as being able to share life long EHRs of patients among different healthcare providers; providing clinical decision support through the use of clinical guidelines which require the interoperability of Electronic Health Record Systems (EHRs), Personal Health Record Systems (PHRs) and wireless medical sensor devices; and enabling secondary use of EHRs for clinical research.

Permission to make digital or hard copies of all or part of this work for personal or classroom use is granted without fee provided that copies are not made or distributed for profit or commercial advantage and that copies bear this notice and the full citation on the first page. To copy otherwise, to republish, to post on servers or to redistribute to lists, requires prior specific permission and/or a fee. Articles from this volume were invited to present their results at The 38th International Conference on Very Large Data Bases, August 27th - 31st 2012, Istanbul, Turkey.

Proceedings of the VLDB Endowment, Vol. 5, No. 12

Copyright 2012 VLDB Endowment 2150-8097/12/08... \$10.00.
Interoperability with regard to a specific task is said to exist between two applications when one application can accept data (including data in the form of a service request) from the other and perform the task in an appropriate and satisfactory manner (as judged by the user of the receiving system) without the need for extra operator intervention [1].

To be interoperable, two applications need to agree on interfaces in each of the three layers of the "interoperability stack": the communication and transport layer; the document layer which involves the format of the exchanged messages and documents as well as the coding systems used, and the business process layer which involves the choreography of the interactions. Bilateral agreements between two applications on these interfaces are not practical because it requires the implementation of a new interface for each application to be communicated with. Therefore standards have been developed to address the various layers in the interoperability stack [7].

However there are a variety of different competing standards that can be used for each layer of the interoperability stack and if applications conform to different standards, the interoperability problem continues. Profiling is used to overcome this challenge by predetermining the combination of the standards to be used and even further restricting them to ensure interoperability.

\section{EHR INTEROPERABILTY}

A survey and analysis of EHR standards are given in [3].

In recent years many regional and national healthcare networks based on Electronic Health Records (EHRs) have been established all over Europe. An example is the National Health Information System of Turkey (NHIS-T) [2] which is a nation-wide infrastructure for sharing patients electronic health records (EHRs). Currently, $98 \%$ of the public hospitals and $60 \%$ of the private and university hospitals are connected to NHIS-T with daily feeds of their patients EHRs. Out of the 74 million citizens of Turkey, electronic healthcare records of 60 million citizens have already been created in NHIS-T.

Another interesting example is the epSOS project [5] for sharing electronic Patient Summary and Prescription/Dispensation documents all over the Europe. Basically, epSOS targets the access to a traveling European citizen's health care data by the health professionals in the European countries he is visiting, so that continuity of care in an informed manner is secured. Started in July 2008, epSOS is composed of 47 beneficiaries from 23 European countries, and currently a few of these countries have enabled epSOS services in real 
environments. Year 2013 and beyond is devoted to full operation by all the participating nations.

\section{PERSONAL HEALTH RECORD (PHR) SYSTEM INTEROPERABILITY}

PHRs are not only electronic repositories of health information controlled or accessed by patients. They are also integrated with a wide variety of healthcare information technology systems including the personal medical devices to obtain the patients instant physiological status; the clinical decision support services for patient-physician shared decision making; and the evidence-based medical sources on the Internet to automatically retrieve data according to the patient context. There are a number of standards addressing the interoperability challenges to communicate with these systems. Therefore, the interoperability standards for the PHR systems can be categorized according to the systems they are communicating with: (i) Electronic Health Record standards, (ii) Personal Medical Device standards, (iii) Clinical Decision Support Services (CDSSs), and (iv) the medical information sources on the Web.

One of the most widely used standards that define PHR summary data is the E2369-05 Standard Specification for Continuity of Care Record (CCR) [10] by the American Society for Testing and Materials (ASTM) International. The IHE XPHR profile, on the other hand, provides an interoperability mechanism to exchange data between PHR systems and the healthcare providers information systems. The EHR-PHR-Medical Device Interoperability standards address how to map the device data obtained through the ISO/IEEE 11073 standards to the healthcare application interfaces. Considering that there are different EHR/PHR content standards and templates, generating a mapping from ISO/IEEE 11073 Domain Information Model to each of them is not very practical.

A recent work [9] addresses this challenge by specializing the HL7 v3 Reference Information Model (RIM) to the medical device domain using the ISO/IEEE 11073 Domain Information Model (DIM) to obtain its Refined Message Information Model (RMIM). The novelty of this approach is that it provides a common denominator for different HL7 v3 RIM based interfaces of various EHR/PHR content models rather than using the bilateral mappings between the device models and different application standards. This facilitates EHR/PHR and personal medical device data interoperability because the concepts are derived from a common RIM through a well-defined refinement process and hence the building blocks of the interfaces are similar, and they can be traceable back to the RIM.

As an example to the use of PHRs for patient self management, the EMPOWER Project [4] aims to support the diabetes patients through a standards-based Patient Empowerment Framework based on Personal Health Record Systems and context-aware personalized action plans.

The PALANTE project [8], on the other hand, focuses on the implementation, scaling up and optimisation of 7 pilot demonstrations based on the concept of secure and user friendly online access by citizens to their medical/health data to empower patients so they will be able to make informed decisions about their health, take an active role in their care and collaborate effectively with their healthcare team thanks to the use of information and communication technologies.

\section{THE SECONDARY USE OF EHRS FOR CLINICAL RESEARCH}

The efficiency with which clinical research studies are conducted affects faster medication innovation and decreases time to market for new drugs. To increase this efficiency, the parties involved in a regulated clinical research study, namely, the sponsor, the clinical investigator and the regulatory body, each with their own software applications, need to exchange data seamlessly. However, currently, the clinical research and the clinical care domains are quite disconnected because each use different standards and terminology systems [6].

The SALUS Semantic Framework [6] addresses these challenges based on semantic mediation, which is a process of matching schemas and mapping attributes and values using semantics.

\section{PRESENTER}

Asuman Dogac is the general manager of SRDC Ltd. She was a full professor at the Computer Engineering Department of the Middle East Technical University till Jan. 2011. SRDC Ltd. is a spin-off of the SRDC research center at the Middle East Technical University.

\section{REFERENCES}

[1] N. Brown and M. Reynolds. Strategy for production and maintenance of standards for interoperability within and between service departments and other healthcare domains. Short Strategic Study CEN/TC 251/N00-047, CEN/TC 251 Health Informatics, Brussels, Belgium, 2000.

[2] A. Dogac, M. Yuksel, and et. al. Electronic Health Record Interoperability as Realized in the Turkish Health Information System. Methods Inf Med, 50(2):140 - 149, 2011.

[3] M. Eichelberg, T. Aden, J. Riesmeier, A. Dogac, and G. B. Laleci. A survey and analysis of Electronic Healthcare Record standards. ACM Comput. Surv., 37:277-315, December 2005.

[4] EMPOWER - Support of Patient Empowerment by an intelligent self-management pathway for patients. http://www.empower-fp7.eu/. Accessed: June 2012.

[5] epSOS - Smart Open Services for European Patients. http://www.epsos.eu/. Accessed: June 2012.

[6] G. B. Laleci, M. Yuksel, and A. Dogac. Providing Semantic Interoperability between Clinical Care and Clinical Research Domains. Submitted for publication.

[7] T. Namli and A. Dogac. Testing Conformance and Interoperability of eHealth Applications. Methods Inf Med, 49(3):281 - 289, 2010.

[8] PALANTE - PAtient Leading and mANaging their healThcare through EHealth. http://palante-project.eu/. Accessed: June 2012.

[9] M. Yuksel and A. Dogac. Interoperability of Medical Device Information and the Clinical Applications: An HL7 RMIM based on the ISO/IEEE 11073 DIM . IEEE Transactions on Information Technology in Biomedicine, 15(4):557 - 566, 2011. 